Homology, Homotopy and Applications, vol.21(2), 2019, pp.345-362

\title{
THE ADAMS-HILTON MODEL AND THE GROUP OF SELF-HOMOTOPY EQUIVALENCES OF A SIMPLY CONNECTED CW-COMPLEX
}

\author{
MAHMOUD BENKHALIFA
}

(communicated by Alexander Mishchenko)

\begin{abstract}
Let $R$ be a principal ideal domain (PID). For a simply connected CW-complex $X$ of dimension $n$, let $Y$ be a space obtained by attaching cells of dimension $q$ to $X, q>n$, and let $A(Y)$ denote an Adams-Hilton model of $Y$. If $\mathcal{E}(A(Y))$ denotes the group of homotopy self-equivalences of $A(Y)$ and $\mathcal{E}_{*}(A(Y))$ its subgroup formed of the elements inducing the identity on $H_{*}(Y, R)$, then we construct two short exact sequences:

$$
\begin{aligned}
\oplus_{i} H_{q}(\Omega X, R) & \longmapsto \mathcal{E}(A(Y)) \rightarrow \Gamma_{n}^{q}, \\
\oplus_{i} H_{q}(\Omega X, R) & \longmapsto \mathcal{E}_{*}(A(Y)) \rightarrow \Pi_{n}^{q},
\end{aligned}
$$

where $i=\operatorname{rank} H_{q}(Y, X ; R), \Pi_{n}^{q}$ is a subgroup of $\mathcal{E}_{*}(A(X))$ and $\Gamma_{n}^{q}$ is a subgroup of $\operatorname{aut}\left(\operatorname{Hom}\left(H_{q}(Y, X ; R)\right)\right) \times \mathcal{E}(A(X))$.
\end{abstract}

\section{Introduction}

Let $R$ be a PID and let $Y$ be a simply connected CW-complex. The Adams-Hilton model of $Y$ is a chain algebra morphism

$$
\Theta_{Y}:(\mathbb{T}(V), \partial) \rightarrow C_{*}(\Omega Y, R)
$$

such that $H_{*}\left(\Theta_{Y}\right): H_{*}(\mathbb{T}(V), \partial) \rightarrow H_{*}(\Omega Y, R)$ is an isomorphism of graded algebras and such that $H_{*}(V, d) \cong H_{*}(Y, R)$ as graded $R$-modules, where $d$ denotes the linear part of the differential $\partial$ induced on the graded module of indecomposables $V$, where $C_{*}(\Omega Y, R)$ denotes the complex of non-degenerate cubic chains equipped with the multiplication induced by composition of loops and where $(\mathbb{T}(V), \partial)$ is the free chain $R$-algebra on the free graded $R$-module $V$. Let $A(Y)$ denote the Adams-Hilton model of the space $Y$.

As is well known, there is a reasonable concept of "homotopy" among chain algebra morphisms (see Section 3), analogous in many respects to the topological notion of homotopy. Consequently, let $\mathcal{E}(A(Y))$ denote the group of homotopy self-equivalences of the chain algebra $A(Y)$.

Received June 9, 2018, revised July 22, 2018, November 14, 2018; published on June 5, 2019. 2010 Mathematics Subject Classification: 55P10.

Key words and phrases: group of homotopy self-equivalences, Adams-Hilton model, Anick model, loop space.

Article available at http://dx.doi.org/10.4310/HHA.2019.v21.n2.a19

Copyright (C) 2019, International Press. Permission to copy for private use granted. 
By the properties of the Adams-Hilton model, it is worth noting that if $\alpha: Y \rightarrow$ $Y$ is a homotopy equivalence, then so is $A(\alpha): A(Y) \rightarrow A(Y)$. Therefore there is a homomorphism of groups $\mathcal{E}(Y) \rightarrow \mathcal{E}(A(Y))$ sending $[\alpha]$ to $[A(\alpha)]$, where $\mathcal{E}(Y)$ is the group of homotopy self-equivalences of $Y$ (see, for example, [11] for more details about this group).

The idea of inserting the group $\mathcal{E}(Y)$ in a short exact sequence of groups of the form $A \longmapsto \mathcal{E}(Y) \rightarrow B$ traces back to the first results on this group in the 1950s. BarcusBarrett [3] gave an exact sequence describing the effect of a single cell attachment $Y=$ $S^{n} \cup_{\alpha} S^{q-1}$ on the group $\mathcal{E}(Y)$. This basic result was refined and extended by later authors including Kahn [13], Oka-Sawashita-Sugawara [14], Benkhalifa-Smith[11] and Benkhalifa $[\mathbf{5}, \mathbf{7}, \mathbf{8}]$. We refer the reader to $[\mathbf{1 5}, \mathbf{1 6}]$ for a comprehensive survey on these results including various exact sequences.

The aim of this paper is to study the effect of cell-attachment on the group $\mathcal{E}(A(X))$. More precisely let $X$ be a simply connected CW-complex of dimension $n$ and let

$$
Y=X \cup_{\alpha}\left(\bigcup_{i \in I} e_{i}^{q}\right)
$$

be the space obtained by attaching cells of dimension $q$ to $X$ by a map $\alpha: \underset{i \in I}{\vee} S^{q-1} \rightarrow$ $X$. Let $\mathcal{E}_{*}(A(Y))$ denote the subgroup of $\mathcal{E}(A(Y))$ consisting of the elements inducing the identity on $H_{*}(Y, R)$. We prove:

Theorem 1. For every $n$ and for every $q>n$, there exist two short exact sequences of groups

$$
\oplus_{i} H_{q}(\Omega X, R) \longmapsto \mathcal{E}(A(Y)) \rightarrow \Gamma_{n}^{q}, \quad \oplus_{i} H_{q}(\Omega X, R) \longmapsto \mathcal{E}_{*}(A(Y)) \rightarrow \Pi_{n}^{q},
$$

where $i=\operatorname{rank} H_{q}(Y, X ; R)$, where $\Gamma_{n}^{q}$ is a subgroup of $\operatorname{aut}\left(\operatorname{Hom}\left(H_{q}(Y, X ; R)\right)\right) \times$ $\mathcal{E}(A(X))$ and $\Pi_{n}^{q}$ is a subgroup of $\mathcal{E}_{*}(A(X))$ (see Definition 4.1)

An analogous problem was previously studied in $[6]$, in terms of the Postnikov decomposition of the rational space $Y$ and by the use of the Sullivan model in rational homotopy theory and it was shown that

Theorem 2 ([6], Corollary 3.3). There exist two exact short sequences:

$$
\begin{aligned}
\operatorname{Hom}\left(\pi_{q}(Y) ; H^{q}\left(Y^{[n]}\right)\right) & \longmapsto \mathcal{E}\left(Y^{[n+1]}\right) \rightarrow D_{n-1}^{n}, \\
\operatorname{Hom}\left(\pi_{q}(Y) ; H^{q}\left(Y^{[n]}\right)\right) & \longmapsto \mathcal{E}_{\#}\left(Y^{[n+1]}\right) \rightarrow \mathrm{G}_{n-1}^{n},
\end{aligned}
$$

where $D_{n}^{q}$ is a subgroup of $\operatorname{aut}\left(\operatorname{Hom}\left(\pi_{q}(Y), \mathbb{Q}\right)\right) \times \mathcal{E}\left(Y^{[n]}\right)$ and where $G_{n}^{q}$ is a subgroup of $\mathcal{E}_{\#}\left(Y^{[n]}\right)$. Here $Y^{[k]}$ denotes the $k^{\text {th }}$ Postnikov section of $Y$ and $\mathcal{E}_{\#}\left(Y^{[n]}\right)$ denotes the subgroup of $\mathcal{E}\left(Y^{[n]}\right)$ consisting of the elements inducing the identity on homotopy groups.

In particular, let $Y$ be a simply connected CW-complex and $\Sigma Y$ the suspension of $Y$. A well-known theorem due to Bott-Samelson [12] asserts that, under the assumption that the homology $H_{*}(Y ; R)$ is a free graded $R$-module, the chain algebra $\left(\mathbb{T}\left(H_{*}(Y ; R)\right), 0\right)$ (with the trivial differential) can be considered as an Adams-Hilton model for the space $\Sigma Y$. Consequently, we prove that the group $\mathcal{E}(A(\Sigma Y))$ is simply 
identified with the group aut $(A(\Sigma Y))$ of the chain algebra automorphisms of $A(\Sigma Y)$ and the subgroup $\mathcal{E}_{*}(A(\Sigma Y))$ is identified with the subgroup aut $(A(\Sigma Y))$ of the chain algebra automorphisms inducing the identity on the graded module $H_{*}(Y, R)$. Moreover, applying Theorem 1, we obtain the following short exact sequences of groups

Theorem 3. Let $Y$ be a simply connected $C W$-complex and $\Sigma Y$ the suspension of $Y$. There exist two exact short sequences

$$
\begin{aligned}
\operatorname{Hom}\left(H_{q+1}(Y ; R), \mathbb{T}_{q}\left(H_{<q}(Y ; R)\right)\right) & \longmapsto \operatorname{aut}(A(\Sigma Y)) \\
& \rightarrow \operatorname{aut}\left(H_{q+1}(Y ; R)\right) \times \operatorname{aut}\left(A\left((\Sigma Y)^{q-1}\right)\right), \\
\operatorname{Hom}\left(H_{q+1}(Y ; R), \mathbb{T}_{q}\left(H_{<q}(Y ; R)\right)\right) \longmapsto & \operatorname{aut}_{*}(A(\Sigma Y)) \rightarrow \operatorname{aut}_{*}\left(A\left((\Sigma Y)^{q-1}\right)\right),
\end{aligned}
$$

where $(\Sigma Y)^{q-1}$ is the $(q-1)$-skeleton of the space $\Sigma Y$.

For instance, using the above short exact sequence we can show the following results

$$
\begin{aligned}
\operatorname{aut}\left(A\left(\mathbb{S}^{n+1} \vee \mathbb{S}^{q+1}\right)\right) & \cong \mathbb{Z}_{2} \times \mathbb{Z}_{2}, \\
\operatorname{aut}_{*}\left(A\left(\mathbb{S}^{n+1} \vee \mathbb{S}^{q+1}\right)\right) & \cong \mathbb{Z}_{2}, \quad \text { if } q \neq 0 \quad(\bmod n), \\
\mathbb{Z} \longmapsto \operatorname{aut}\left(A\left(\mathbb{S}^{n+1} \vee \mathbb{S}^{q+1}\right)\right) & \rightarrow \mathbb{Z}_{2} \times \mathbb{Z}_{2}, \\
\mathbb{Z} \longmapsto \operatorname{aut}_{*}\left(A\left(\mathbb{S}^{n+1} \vee \mathbb{S}^{q+1}\right)\right) & \rightarrow \mathbb{Z}_{2}, \quad \text { if } q \equiv(\bmod n) .
\end{aligned}
$$

Moreover, let $R \subseteq \mathbb{Q}$ be a subring with least non-invertible prime $p$. Using the Anick model theory $[\mathbf{1}, \mathbf{2}]$, if $X$ is an $r$-connected CW-complex of dimension $n+1$ and $n<q \leqslant k$, where $k<\min (r+2 p-3, r p-1)$, then we prove

Theorem 4. Let $Y$ be the space in (1). The homomorphisms

$$
\mathcal{E}\left(Y_{R}\right) \rightarrow \mathcal{E}\left(A\left(Y_{R}\right)\right), \quad \mathcal{E}_{*}\left(Y_{R}\right) \rightarrow \mathcal{E}_{*}\left(A\left(Y_{R}\right)\right)
$$

are injective, where $Y_{R}$ denotes the $R$-localization of $Y$.

The paper is organized as follows. In Section 2 we recall briefly the notion of the Adams-Hilton model associated to a given simply connected space $Y$ and the BottSamelson theorem concerning the Adams-Hilton model of the space $\Sigma Y$. In Section 3 we establish Theorem 3, and in Section 4 we recall the notion of homotopy between chain algebra morphisms and we prove Theorem 2 and some of its corollaries.

\section{Adams-Hilton model and Bott-Samelson theorem}

Given a simply connected CW-complex $Y$. The Adams-Hilton model of $Y$ is a chain algebra morphism

$$
\Theta_{Y}:(\mathbb{T}(V), \partial) \rightarrow C_{*}(\Omega Y, R)
$$

such that

$$
H_{*}\left(\Theta_{Y}\right): H_{*}(\mathbb{T}(V), \partial) \rightarrow H_{*}(\Omega Y, R)
$$

is an isomorphism of graded algebras and such that

$$
H_{i-1}(V, d) \cong H_{i}(Y, R),
$$


as graded modules. Here $C_{*}(\Omega Y, R)$ denotes the complex of non-degenerate cubic chains equipped with the multiplication induced by composition of loops and $d: V \rightarrow$ $V$ is the linear part of the differential $\partial$ defined by

$$
\partial(v)-d(v) \in \mathbb{T}^{\geqslant 2}(V),
$$

where $\mathbb{T}^{\geqslant 2}(V)$ is the graded $R$-module of decomposable elements, i.e., the elements of $\mathbb{T}(V)$ of length $\geqslant 2$. We denote by $A(Y)$ the chain algebra $(\mathbb{T}(V), \partial)$.

Let $\Sigma Y$ denote the suspension of $Y$. If the map $\sigma: Y \rightarrow \Omega \Sigma Y$ is the adjoint of $i d_{\Sigma Y}$, then it induces a homomorphism of graded modules $\sigma_{*}: H_{*}(Y ; R) \rightarrow H_{*}(\Omega \Sigma Y ; R)$ which can be extended, by virtue of the universal property of the free chain algebra, to a homomorphism

$$
\mathbb{T}\left(\sigma_{*}\right): \mathbb{T}\left(H_{*}(Y ; R)\right) \rightarrow H_{*}(\Omega \Sigma Y ; R)
$$

of graded algebras.

A theorem, due to Bott and Samelson [12], asserts that under the assumption that the homology $H_{*}(Y ; R)$ is a free graded $R$-module, $\mathbb{T}\left(\sigma_{*}\right)$ is an isomorphism of $R$ algebras. Therefore the chain algebra $\left(\mathbb{T}\left(H_{*}(Y ; R)\right), 0\right)$, with the trivial differential, can be considered as an Adams-Hilton model for the space $\Sigma Y$, i.e.,

$$
A(\Sigma Y)=\left(\mathbb{T}\left(H_{*}(Y ; R)\right), 0\right) .
$$

By the properties of the Adams-Hilton model we derive

$$
H_{*}(\Omega \Sigma Y ; R)=H_{*}(A(\Sigma Y))=\mathbb{T}\left(H_{*}(Y ; R)\right) .
$$

Remark 2.1. It is important to mention here that as the graded $R$-module $H_{*}(Y ; R)$ is assumed to be free, the two relations (2) and (3) imply that the Adams-Hilton model $(\mathbb{T}(V), 0)$ of $\Sigma Y$ satisfies

$$
V_{i} \cong H_{i-1}(Y ; R), \quad \forall i \geqslant 2 .
$$

\section{The group of graded algebra automorphisms of the tensor algebra $\mathbb{T}(V)$}

Let $\mathbb{T}\left(V_{q} \oplus V_{\leqslant n}\right)$, where $q>n$, be a tensor algebra (considered as 1-connected chain algebra with trivial differential). Let us denote by $\operatorname{aut}\left(\mathbb{T}\left(V_{q} \oplus V_{\leqslant n}\right)\right)$ the group of chain (graded) algebra automorphisms of $\mathbb{T}\left(V_{q} \oplus V_{\leqslant n}\right)$.

If $\alpha \in \operatorname{aut}\left(\mathbb{T}\left(V_{q} \oplus V_{\leqslant n}\right)\right)$, then it induces the following homomorphism

$$
\alpha_{k}: V_{k} \rightarrow V_{k} \oplus \mathbb{T}_{k}\left(V_{\leqslant n}\right), \quad k \leqslant q
$$

so define $\widetilde{\alpha}_{k}: V_{k} \rightarrow V_{k}$ such that $\alpha(v)-\widetilde{\alpha}_{k}(v) \in \mathbb{T}_{k}\left(V_{\leqslant n}\right)$. Clearly $\widetilde{\alpha}_{k}$ is an automorphism of $V_{k}$. Hence denote by aut $*\left(\mathbb{T}\left(V_{q} \oplus V_{\leqslant n}\right)\right)$ the subgroup of aut $\left(\mathbb{T}\left(V_{q} \oplus V_{\leqslant n}\right)\right)$ consisting of the elements $\alpha$ such that $\widetilde{\alpha}_{k}=i d$ for all $k \leqslant q$.

The aim of this section is to establish the following theorem:

Theorem 3.1. If $\mathbb{T}(V)$ is a 1-connected free graded tensor algebra, then we have the following two short exact sequences of groups

$$
\begin{array}{r}
\operatorname{Hom}\left(V_{q}, \mathbb{T}_{q}\left(V_{\leqslant n}\right)\right) \longmapsto \operatorname{aut}\left(\mathbb{T}\left(V_{q} \oplus V_{\leqslant n}\right)\right) \rightarrow \operatorname{aut}\left(V_{q}\right) \times \operatorname{aut}\left(\mathbb{T}\left(V_{\leqslant n}\right)\right), \\
\operatorname{Hom}\left(V_{q}, \mathbb{T}_{q}\left(V_{\leqslant n}\right)\right) \longmapsto \operatorname{aut}_{*}\left(\mathbb{T}\left(V_{q} \oplus V_{\leqslant n}\right)\right) \rightarrow \operatorname{aut}_{*}\left(\mathbb{T}\left(V_{\leqslant n}\right)\right) .
\end{array}
$$


Proof. Let $\left(\mathbb{T}\left(V_{q} \oplus V_{\leqslant n}\right)\right)$, where $q>n$, be a free graded tensor algebra. Define the map

$$
\mathrm{g}: \operatorname{aut}\left(\mathbb{T}\left(V_{q} \oplus V_{\leqslant n}\right)\right) \rightarrow \operatorname{aut}\left(V_{q}\right) \times \operatorname{aut}\left(\mathbb{T}\left(V_{\leqslant n}\right)\right)
$$

by setting:

$$
\mathrm{g}(\alpha)=\left(\widetilde{\alpha}_{q}, \alpha_{n}\right),
$$

where $\widetilde{\alpha}_{q}: V_{q} \rightarrow V_{q}$ is as above and where $\alpha_{n}$ is the restriction of $\alpha$ to $\mathbb{T}\left(V_{\leqslant n}\right)$.

It is easy to see that $\mathrm{g}$ is a surjective morphism of groups. Indeed, let $(\xi, \gamma) \in$ $\operatorname{aut}\left(V_{q}\right) \times \operatorname{aut}\left(\mathbb{T}\left(V_{\leqslant n}\right)\right)$. Define $\alpha: \mathbb{T}\left(V_{q} \oplus V_{\leqslant n}\right) \rightarrow \mathbb{T}\left(V_{q} \oplus V_{\leqslant n}\right)$ by setting:

$$
\alpha(v)=\xi(v) \quad \text { and } \quad \alpha=\gamma \text { on } V_{\leqslant n} .
$$

Clearly we have $\widetilde{\alpha}_{q}=\xi$. Hence using (6) we derive $\mathrm{g}(\alpha)=(\xi, \gamma)$.

Finally, the following relations

$$
\mathrm{g}\left(\alpha \circ \alpha^{\prime}\right)=\left(\widetilde{\alpha \circ \alpha^{\prime}}{ }_{q}, \alpha_{n} \circ \alpha_{n}^{\prime}\right)=\left(\widetilde{\alpha}_{q}, \alpha_{n}\right) \circ\left({\widetilde{\alpha^{\prime}}}_{q}, \alpha_{n}^{\prime}\right)=\mathrm{g}(\alpha) \circ \mathrm{g}\left(\alpha^{\prime}\right)
$$

assure that $\mathrm{g}$ is a homomorphism of groups.

Consequently, we obtain the following short exact sequence of groups

$$
\operatorname{ker} \mathrm{g} \longmapsto \operatorname{aut}\left(\mathbb{T}\left(V_{q} \oplus V_{\leqslant n}\right), 0\right) \stackrel{\mathrm{g}}{\rightarrow} \operatorname{aut}\left(V_{q}\right) \times \operatorname{aut}\left(\mathbb{T}\left(V_{\leqslant n}\right)\right) .
$$

Next let us determine ker g. By (6) we can write:

$$
\text { ker } \mathrm{g}=\left\{\alpha \in \operatorname{aut}\left(\mathbb{T}\left(V_{q} \oplus V_{\leqslant n}\right)\right) \mid \widetilde{\alpha}_{q}=i d_{V_{q}}, \alpha_{n}=i d_{\mathbb{T}\left(V_{\leqslant n}\right)}\right\},
$$

therefore for every $\alpha \in$ ker g we have:

$$
\begin{aligned}
\alpha(v) & =v+z_{v}, \quad z_{v} \in \mathbb{T}_{q}\left(V_{\leqslant n}\right), \\
\alpha_{n} & =i d_{\mathbb{T}\left(V_{\leqslant n}\right)} .
\end{aligned}
$$

So define the map $\Psi$ : $\operatorname{ker} g \rightarrow \operatorname{Hom}\left(V_{q}, \mathbb{T}_{q}\left(V_{\leqslant n}\right)\right)$ by setting

$$
\Psi(\alpha): V_{q} \rightarrow \mathbb{T}_{q}\left(V_{\leqslant n}\right), \quad \Psi(\alpha)(v)=z_{v} .
$$

On the one hand the relations (9) and (10) imply that

$$
\alpha \circ \alpha^{\prime}(v)=\alpha\left(v+z_{v}^{\prime}\right)=v+z_{v}+z_{v}^{\prime}
$$

hence $\Psi\left(\alpha \circ \alpha^{\prime}\right)(v)=z_{v}+z_{v}^{\prime}$. On the other hand we have

$$
\left(\Psi(\alpha)+\Psi\left(\alpha^{\prime}\right)\right)(v)=\Psi(\alpha)(v)+\Psi\left(\alpha^{\prime}\right)(v)=z_{v}+z_{v}^{\prime} .
$$

Therefore $\Psi\left(\alpha \circ \alpha^{\prime}\right)=\Psi(\alpha)+\Psi\left(\alpha^{\prime}\right)$ implying that $\Psi$ is a homomorphism of groups.

Now let $\alpha \in \operatorname{ker} \Psi$, then $\Psi(\alpha)=0$ implying that $\Psi(\alpha)(v)=z_{v}=0$, and according to (9), it follows that $\alpha=i d$. Hence $\Psi$ is injective. Finally, let $f \in \operatorname{Hom}\left(V_{q}, \mathbb{T}_{q}\left(V_{\leqslant n}\right)\right)$, and define

$$
\alpha(v)=v+f(v), \quad \alpha_{n}=i d_{\mathbb{T}\left(V_{\leqslant n}\right)} .
$$

By definition (10) we have $\Psi(\alpha)(v)=f(v)$, so $\Psi(\widetilde{\gamma})=f$. It follows that $\Psi$ is surjective, consequently $\Psi$ is an isomorphism of groups. 
Summarizing the short exact sequence (7) becomes

$$
\operatorname{Hom}\left(V_{q}, \mathbb{T}_{q}\left(V_{\leqslant n}\right)\right) \longmapsto \operatorname{aut}\left(\mathbb{T}\left(V_{q} \oplus V_{\leqslant n}\right)\right) \stackrel{\mathrm{g}}{\rightarrow} \operatorname{aut}\left(V_{q}\right) \times \operatorname{aut}\left(\mathbb{T}\left(V_{\leqslant n}\right)\right) .
$$

Next let $\hat{g}$ denote the restriction of the homomorphism $g$ to the subgroup aut $_{*}\left(\mathbb{T}\left(V_{q} \oplus V_{\leqslant n}\right)\right)$. As aut $\left(\mathbb{T}\left(V_{q} \oplus V_{\leqslant n}\right)\right)$ is formed by the elements $\alpha$ such that $\widetilde{\alpha}_{k}=i d$ for all $k \leqslant q$, it follows that

$$
\hat{\mathrm{g}}(\alpha)=\left(i d_{V_{q}}, \alpha_{n}\right), \quad \alpha_{n} \in \mathrm{aut}_{*}\left(\mathbb{T}\left(V_{\leqslant n}\right)\right) .
$$

Hence we define $\widetilde{\mathrm{g}}: \operatorname{aut}_{*}\left(\mathbb{T}\left(V_{q} \oplus V_{\leqslant n}\right)\right) \rightarrow \operatorname{aut}_{*}\left(\mathbb{T}\left(V_{\leqslant n}\right)\right)$ by $\widetilde{\mathrm{g}}(\alpha)=\alpha_{n}$. The map $\widetilde{g}$ is a surjective homomorphism. Indeed, if $\gamma \in \operatorname{aut}_{*}\left(\mathbb{T}\left(V_{\leqslant n}\right)\right)$, then $\left(i d_{V_{q}}, \gamma\right) \in \operatorname{aut}\left(V_{q}\right) \times$ $\operatorname{aut}\left(\mathbb{T}\left(V_{\leqslant n}\right)\right)$. As the homomorphism $g$ is surjective, there exists $\alpha \in \operatorname{aut}\left(\mathbb{T}\left(V_{\leqslant n}\right)\right)$ such that $\mathrm{g}(\alpha)=\left(i d_{V_{q}}, \gamma\right)$. Using $(6)$ we deduce that

$$
\left(i d_{V_{q}}, \gamma\right)=\left(\widetilde{\alpha}_{q}, \alpha_{n}\right)
$$

implying that $\alpha \in \operatorname{aut}_{*}\left(\mathbb{T}\left(V_{\leqslant n}\right)\right)$ and $\widetilde{g}(\alpha)=\gamma$.

Now from (8) we have ker $\mathrm{g}=\operatorname{ker} \widetilde{\mathrm{g}}$ and since ker $\mathrm{g} \cong \operatorname{Hom}\left(V_{q}, \mathbb{T}_{q}\left(V_{\leqslant n}\right)\right)$, we obtain the following short exact sequence

$$
\operatorname{Hom}\left(V_{q}, \mathbb{T}_{q}\left(V_{\leqslant n}\right)\right) \longmapsto \operatorname{aut}_{*}\left(\mathbb{T}\left(V_{q} \oplus V_{\leqslant n}\right)\right) \rightarrow \operatorname{aut}_{*}\left(\mathbb{T}\left(V_{\leqslant n}\right)\right) .
$$

Corollary 3.2. If $\Sigma Y$ is a simply connected space of dimension $q+1$ and $X=(\Sigma Y)^{q}$ denotes the $q$ skeleton of $\Sigma Y$, then the following short sequence of groups is exact:

$$
\begin{aligned}
\operatorname{Hom}\left(H_{q+1}(Y ; R), \mathbb{T}_{q}\left(H_{<q}(Y ; R)\right)\right) & \mapsto \operatorname{aut}(A(\Sigma Y)) \\
& \rightarrow \operatorname{aut}\left(H_{q+1}(Y ; R)\right) \times \operatorname{aut}\left(A\left((\Sigma Y)^{q-1}\right)\right), \\
\operatorname{Hom}\left(H_{q+1}(Y ; R), \mathbb{T}_{q}\left(H_{<q}(Y ; R)\right)\right) & \longmapsto \operatorname{aut}_{*}(A(\Sigma Y)) \rightarrow \operatorname{aut}_{*}\left(A\left((\Sigma Y)^{q-1}\right)\right) .
\end{aligned}
$$

Proof. First the Adams-Hilton of the space $\Sigma Y$ is of the form $\mathbb{T}\left(V_{q} \oplus V_{\leqslant q-1}\right)$ with trivial differential. Next we derive the two sequences in (11) by applying Theorem 3.1 and using the relations (2), (3) and (4).

Corollary 3.3. Let $V_{q}=\left\{v_{q}\right\}$ be the free $R$-module of rank 1 and let $\mathbb{T}\left(V_{q} \oplus V_{\leqslant n}\right)$, where $q>n$, be a free tensor algebra. Then the following short sequence of groups is exact:

$$
\begin{aligned}
\mathbb{T}_{q}\left(V_{\leqslant n}\right) & \longmapsto \operatorname{aut}\left(\mathbb{T}\left(V_{q} \oplus V_{\leqslant n}\right)\right) \rightarrow \operatorname{aut}(R) \times \operatorname{aut}\left(\mathbb{T}\left(V_{\leqslant n}\right)\right), \\
\mathbb{T}_{q}\left(V_{\leqslant n}\right) & \longmapsto \operatorname{aut}_{*}\left(\mathbb{T}\left(V_{q} \oplus V_{\leqslant n}\right)\right) \rightarrow \operatorname{aut}_{*}\left(\mathbb{T}\left(V_{\leqslant n}\right)\right) .
\end{aligned}
$$

Proof. The sequence (12) can be deduced from the exact sequence (5) by observing that $V_{q}=\left\{v_{q}\right\} \cong R$, so $\operatorname{Hom}\left(V_{q}, \mathbb{T}_{q}\left(V_{\leqslant n}\right)\right) \cong \mathbb{T}_{q}\left(V_{\leqslant n}\right)$ and $\operatorname{aut}\left(V_{q}\right) \cong \operatorname{aut}(R)$.

As an illustration of Corollary 3.3 we give the following example:

Example 3.4. Let $R=\mathbb{Z}$ and let $X=\mathbb{S}^{n+1}$ be the sphere of dimension $n+1$. Let $Y=\mathbb{S}^{n+1} \vee \mathbb{S}^{q+1}$, where $q>n$ and where the $q+1$-cell is trivially attached to $\mathbb{S}^{n+1}$.

Recall that $A(\Sigma Y)$ (respectively $A(\Sigma Y)$ ) denotes the Adams-Hilton model of the suspension of the space $Y$ (respectively of $X$ (see 2)) and let aut $(A(\Sigma Y)$ ) (respectively aut $(A(\Sigma Y)))$ denote the group of graded automorphisms of the free tensor algebra $A(\Sigma Y)$ (respectively $A(\Sigma X)$ ). 
The Adams-Hilton models of $S^{n+1}=\Sigma S^{n}$ and $Y=\mathbb{S}^{n+1} \vee \mathbb{S}^{q+1}$ are, respectively,

$$
\begin{aligned}
A\left(S^{n+1}\right) & =\mathbb{T}\left(H_{*}\left(S^{n} ; \mathbb{Z}\right)\right), \\
A\left(\mathbb{S}^{n+1} \vee \mathbb{S}^{q+1}\right) & =A\left(\Sigma\left(\mathbb{S}^{n} \vee \mathbb{S}^{q}\right)\right) \cong \mathbb{T}\left(H_{*}\left(S^{n} \vee \mathbb{S}^{q}\right) ; \mathbb{Z}\right)
\end{aligned}
$$

Recall that

$$
H_{q}\left(S^{n} ; \mathbb{Z}\right)= \begin{cases}0, & \text { if } q \neq n \\ \mathbb{Z}, & \text { if } q=n\end{cases}
$$

and

$$
H_{*}\left(S^{n} \vee \mathbb{S}^{q} ; \mathbb{Z}\right)=H_{*}\left(S^{n} ; \mathbb{Z}\right) \oplus H_{*}\left(\mathbb{S}^{q} ; \mathbb{Z}\right)
$$

Define the graded abelian group $V_{q} \oplus V_{\leqslant n}$ by

$$
V_{q} \cong H_{q}\left(\mathbb{S}^{q} ; \mathbb{Z}\right) \cong \mathbb{Z}, \quad V_{n} \cong H_{n}\left(\mathbb{S}^{n} ; \mathbb{Z}\right) \cong \mathbb{Z}, \quad V_{i}=0, \quad i \leqslant n-1 .
$$

Therefore we obtain

$$
A\left(S^{n+1}\right)=\mathbb{T}\left(V_{\leqslant n}\right), \quad A\left(\mathbb{S}^{n+1} \vee \mathbb{S}^{q+1}\right)=A\left(\Sigma\left(\mathbb{S}^{n} \vee \mathbb{S}^{q}\right)\right) \cong \mathbb{T}\left(V_{q} \oplus V_{\leqslant n} ; \mathbb{Z}\right)
$$

Applying Corollary 3.3 we get

$$
\mathbb{T}_{q}\left(H_{*}\left(S^{n} ; \mathbb{Z}\right)\right) \longmapsto \operatorname{aut}\left(A\left(\mathbb{S}^{n+1} \vee \mathbb{S}^{n+2}\right)\right) \rightarrow \operatorname{aut}(\mathbb{Z}) \times \operatorname{aut}\left(\mathbb{T}\left(H_{*}\left(S^{n} ; \mathbb{Z}\right)\right)\right) .
$$

Let us compute aut $\left(\mathbb{T}\left(H_{*}\left(S^{n} ; \mathbb{Z}\right)\right)\right)$. Indeed, we have

$$
\operatorname{aut}\left(\mathbb{T}\left(H_{*}\left(S^{n} ; \mathbb{Z}\right)\right)\right)=\operatorname{aut}\left(\mathbb{T}\left(V_{\leqslant n}\right)\right)=\operatorname{aut}\left(\mathbb{T}\left(V_{n} \oplus V_{\leqslant n-1}\right)\right) .
$$

Applying Corollary 3.3 again it follows that

$$
\mathbb{T}_{q}\left(V_{\leqslant n-1}\right) \longmapsto \operatorname{aut}\left(\mathbb{T}\left(V_{n} \oplus V_{\leqslant n-1}\right)\right) \rightarrow \operatorname{aut}\left(V_{n}\right) \times \operatorname{aut}\left(\mathbb{T}\left(H_{*}\left(V_{\leqslant n-1}\right)\right)\right)
$$

and taking into account that $V_{\leqslant n-1}=0$ we obtain

$$
\operatorname{aut}\left(\mathbb{T}\left(V_{n} \oplus V_{\leqslant n-1}\right)\right) \cong \operatorname{aut}\left(V_{n}\right) .
$$

Hence

$$
\operatorname{aut}\left(\mathbb{T}\left(H_{*}\left(S^{n} ; \mathbb{Z}\right)\right)\right) \cong \operatorname{aut}\left(V_{n}\right) \cong \operatorname{aut}(\mathbb{Z})=\mathbb{Z}_{2} .
$$

Consequently, the exact sequence (13) becomes

$$
\mathbb{T}_{q}\left(H_{*}\left(S^{n} ; \mathbb{Z}\right)\right) \longmapsto \operatorname{aut}\left(A\left(\mathbb{S}^{n+1} \vee \mathbb{S}^{q+1}\right)\right) \rightarrow \mathbb{Z}_{2} \times \mathbb{Z}_{2}
$$

But we have

$$
\mathbb{T}_{q}\left(H_{*}\left(S^{n} ; \mathbb{Z}\right)\right)=\left\{\begin{array}{lll}
0, & \text { if } q \neq \equiv 0 & (\bmod n) \\
\mathbb{Z}, & \text { if } q \equiv 0 & (\bmod n)
\end{array}\right.
$$

implying

$$
\begin{aligned}
\operatorname{aut}\left(A\left(\mathbb{S}^{n+1} \vee \mathbb{S}^{q+1}\right)\right) \cong \mathbb{Z}_{2} \times \mathbb{Z}_{2}, & \text { if } q \neq 0 \quad(\bmod n), \\
\mathbb{Z} \longmapsto \operatorname{aut}\left(A\left(\mathbb{S}^{n+1} \vee \mathbb{S}^{q+1}\right)\right) & \rightarrow \mathbb{Z}_{2} \times \mathbb{Z}_{2}, \quad \text { if } q \equiv 0 \quad(\bmod n) .
\end{aligned}
$$

Finally, a similar computation shows that

$$
\begin{aligned}
\operatorname{aut}_{*}\left(A\left(\mathbb{S}^{n+1} \vee \mathbb{S}^{q+1}\right)\right) \cong \mathbb{Z}_{2}, & \text { if } q \neq 0 \quad(\bmod n), \\
\mathbb{Z} \longmapsto \operatorname{aut}_{*}\left(A\left(\mathbb{S}^{n+1} \vee \mathbb{S}^{q+1}\right)\right) \rightarrow \mathbb{Z}_{2}, & \text { if } q \equiv 0 \quad(\bmod n) .
\end{aligned}
$$

Notice that $\operatorname{aut}_{*}\left(A\left(\mathbb{S}^{n+1} \vee \mathbb{S}^{q+1}\right)\right)$ is a normal subgroup of $\operatorname{aut}\left(A\left(\mathbb{S}^{n+1} \vee \mathbb{S}^{q+1}\right)\right)$ and 
in the two cases the quotient group is

$$
\frac{\operatorname{aut}\left(A\left(\mathbb{S}^{n+1} \vee \mathbb{S}^{q+1}\right)\right)}{\operatorname{aut}_{*}\left(A\left(\mathbb{S}^{n+1} \vee \mathbb{S}^{q+1}\right)\right)} \cong \mathbb{Z}_{2}
$$

In the second part of this paper we shall generalize the above results to the case when the differential given in $(\mathbb{T}(V), \partial)$ is not necessarily trivial. For this purpose we need the notion of homotopy between chain algebra morphisms which is analogous in many respects to the topological notion of homotopy.

\section{The group of homotopy self-equivalences of chain algebra morphisms}

\subsection{Homotopy of chain algebra morphisms}

(See [4] page 48 for more details). Let $(\mathbb{T}(V), \partial)$ be a 1-connected free chain algebra. Define the free algebra $\mathbb{T}\left(V^{\prime} \oplus V^{\prime \prime} \oplus s V\right)$, where $V^{\prime}, V^{\prime \prime}$ are two isomorphic copies of $V$ and $s V$ is the (de)suspension of $V$. Then we define:

$$
i^{\prime}, i^{\prime \prime}: \mathbb{T}(V) \rightarrow \mathbb{T}\left(V^{\prime} \oplus V^{\prime \prime} \oplus s V\right), \quad i^{\prime}(v)=v^{\prime}, \quad i^{\prime \prime}(v)=v^{\prime \prime},
$$

where $v^{\prime} \in V^{\prime}, v^{\prime \prime} \in V^{\prime \prime}$ are the two elements corresponding to $v \in V$. Now define $S: \mathbb{T}(V) \rightarrow \mathbb{T}\left(V^{\prime} \oplus V^{\prime \prime} \oplus s V\right)$, of degree 1 , as the unique graded module homomorphism which satisfies the following two conditions

$S(v)=s v, \quad S(x . y)=S(x) \cdot\left(i^{\prime \prime}(y)+(-1)^{|x|} i^{\prime}(x)\right) S(y), \quad \forall v \in V$ and $x, y \in \mathbb{T}(V)$.

Next we define the differential $D$ on $\mathbb{T}\left(V^{\prime} \oplus V^{\prime \prime} \oplus s V\right)$ by setting

$$
D(s v)=v^{\prime \prime}-v^{\prime}-S(\partial v), \quad D\left(v^{\prime}\right)=i^{\prime}(\partial v), \quad D\left(v^{\prime \prime}\right)=i^{\prime \prime}(\partial v) .
$$

$\left(\mathbb{T}\left(V^{\prime} \oplus V^{\prime \prime} \oplus s V\right), D\right)$ is called the cylinder chain algebra of $(\mathbb{T}(V), \partial)$.

Definition 4.1. A homotopy between two chain algebra morphisms $\alpha_{1}, \alpha_{2}:(\mathbb{T}(V), \partial)$ $\rightarrow(\mathbb{T}(V), \partial)$ is a chain algebra morphism

$$
F:\left(\mathbb{T}\left(V^{\prime} \oplus V^{\prime \prime} \oplus s V\right), D\right) \rightarrow(\mathbb{T}(V), \partial)
$$

such that $F \circ i^{\prime}(v)=F\left(v^{\prime}\right)=\alpha_{1}(v)$ and $F \circ i^{\prime \prime}(v)=F\left(v^{\prime \prime}\right)=\alpha_{2}(v)$.

Definition 4.2. A chain algebra morphism $\alpha_{1}:(\mathbb{T}(V), \partial) \rightarrow(\mathbb{T}(V), \partial)$ is called a self-homotopy equivalence, if there exists a chain algebra morphism $\alpha_{2}:(\mathbb{T}(V), \partial) \rightarrow$ $(\mathbb{T}(V), \partial)$ such that $\alpha_{1} \circ \alpha_{2}$ and $\alpha_{2} \circ \alpha_{1}$ are homotopic to the identity.

Definition 4.3. Let $\mathcal{E}(\mathbb{T}(V))$ denote the group of homotopy classes of self-homotopy equivalences of $(\mathbb{T}(V), \partial)$, under composition of chain algebra morphisms, and let $\mathcal{E}_{*}(\mathbb{T}(V))$ denote the subgroup consisting of those elements which induce the identity on the graded module of indecomposable $V_{*}$.

Thereafter we will need the following lemma:

Lemma 4.4. Let $q>n$, let $V=V_{q} \oplus V_{\leqslant n}$ and let $\alpha_{1}, \alpha_{2}:(\mathbb{T}(V), \partial) \rightarrow(\mathbb{T}(V), \partial)$ be two chain algebra morphisms satisfying:

$$
\alpha_{1}(v)=v+z_{1}, \quad \alpha_{2}(v)=v+z_{2} \quad \text { on } V_{q} \quad \text { and } \quad \alpha=\alpha^{\prime}=\mathrm{id} \quad \text { on } V_{\leqslant n} .
$$

Assume that $z_{1}-z_{2}=\partial(u)$, where $u \in \mathbb{T}_{q+1}(V)$. Then $\alpha_{1}$ and $\alpha_{2}$ are homotopic. 
Proof. Define $F$ by setting

$$
\begin{aligned}
& F\left(v^{\prime}\right)=v+z_{1}, \quad F\left(v^{\prime \prime}\right)=v+z_{2} \quad \text { and } \quad F(s v)=u \quad \text { for } \quad v \in V^{q} \text {, } \\
& F\left(v^{\prime}\right)=v, \quad F\left(v^{\prime \prime}\right)=v \quad \text { and } \quad F(s v)=0 \text { for } v \in V^{\leqslant n} \text {, }
\end{aligned}
$$

then $F$ is the required homotopy.

We start with the following remarks.

Remark 4.5. If $(\mathbb{T}(V), 0)$ is a 1-connected free chain algebra with trivial differential, then the notion of homotopy is simply the equality. Indeed, let $\alpha_{1}, \alpha_{2}:(\mathbb{T}(V), 0) \rightarrow$ $(\mathbb{T}(V), 0)$ be two chain algebra morphisms and assume that they are homotopic. By Definition 4.1 there exists a chain algebra morphism

$$
F:\left(\mathbb{T}\left(V^{\prime} \oplus V^{\prime \prime} \oplus s V\right), D\right) \rightarrow(\mathbb{T}(V), 0)
$$

such that

$$
F \circ i^{\prime}(v)=F\left(v^{\prime}\right)=\alpha_{1}(v), \quad F \circ i^{\prime \prime}(v)=F\left(v^{\prime \prime}\right)=\alpha_{2}(v) .
$$

As the differential $\partial$ is trivial and $F$ is a chain algebra, it follows that

$$
F \circ D=0 \text {, }
$$

moreover, the relations (14) become

$$
D(s v)=v^{\prime \prime}-v^{\prime}, \quad D\left(v^{\prime}\right)=0, \quad D\left(v^{\prime \prime}\right)=0 .
$$

Therefore

$$
0=F \circ D(s v)=F\left(v^{\prime \prime}\right)-F\left(v^{\prime}\right) .
$$

Finally, according to $(15)$ we deduce that $\alpha_{1}(v)=\alpha_{2}(v)$.

Remark 4.6. Let $(\mathbb{T}(V), 0)$ be a 1-connected free chain algebra with trivial differential. By virtue of Remark 4.5 we derive that the group $\mathcal{E}(\mathbb{T}(V))$ is identified with the group aut $(\mathbb{T}(V))$ and $\mathcal{E}_{*}(\mathbb{T}(V))$ is identified with the subgroup aut $*(\mathbb{T}(V))$ introduced in the previous section.

\subsection{The graded homomorphism $b_{*}$ and the groups $\mathcal{D}_{n}^{q}$}

Definition 4.7. Let $\left(\mathbb{T}\left(V_{q} \oplus V_{\leqslant n}\right), \partial\right)$ be a 1-connected chain algebra where $q>n$. We define the homomorphism $b_{q}: V_{q} \rightarrow H_{q-1}\left(\mathbb{T}\left(V_{\leqslant n}\right)\right)$ by setting:

$$
b_{q}(v)=[\partial(v)] .
$$

Here $[\partial(v)]$ denotes the homology class of $\partial(v) \in \mathbb{T}_{q-1}\left(V_{\leqslant n}\right)$.

For every 1-connected chain algebra $\left(\mathbb{T}\left(V_{q} \oplus V_{\leqslant n}\right), \partial\right)$, the homomorphism $b_{q}$ is natural. Namely if $[\alpha] \in \mathcal{E}\left(\mathbb{T}\left(V_{q} \oplus V_{\leqslant n}\right)\right)$, then the following diagram commutes:

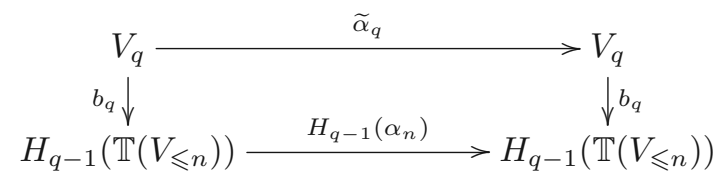

where

$$
\widetilde{\alpha}:\left(V_{q} \oplus V_{\leqslant n}, d\right) \rightarrow\left(V_{q} \oplus V_{\leqslant n}, d\right)
$$

is the graded homomorphism induced by $\alpha$ on the chain complex of indecomposables 
and where $\alpha_{n}:\left(\mathbb{T}\left(V_{\leqslant n}\right), \partial\right) \rightarrow\left(\mathbb{T}\left(V_{\leqslant n}\right), \partial\right)$ is the restriction of $\alpha$. Here $d$ denotes the linear part of the differential $\partial$ defined by the relation

$$
\partial-d: V_{n+1} \rightarrow \mathbb{T}_{n}^{\geqslant 2}(V)
$$

Definition 4.8. Given a 1-connected chain algebra $\left(\mathbb{T}\left(V_{q} \oplus V_{\leqslant n}\right), \partial\right)$ where $q>n$, set $V=V_{q} \oplus V_{\leqslant n}$. Let $\mathcal{D}_{n}^{q}$ be the subset of aut $\left(V_{q}\right) \times \mathcal{E}\left(\mathbb{T}\left(V_{\leqslant n}\right)\right)$ consisting of the couples $(\xi,[\alpha])$ making the following diagram commute

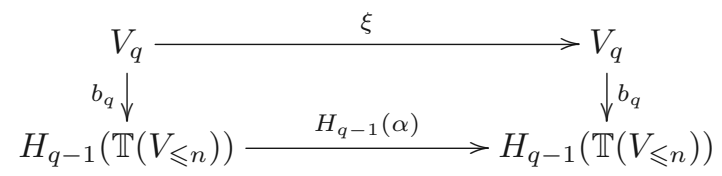

Clearly $\mathcal{D}_{n}^{q}$ is a subgroup of $\operatorname{aut}\left(V_{q}\right) \times \mathcal{E}\left(\mathbb{T}\left(V_{\leqslant n}\right)\right)$.

Remark 4.9. If $(\mathbb{T}(V), 0)$ is a 1-connected free chain algebra with trivial differential, then according to the relation (16), the homomorphism $b_{q}$ given in the diagram (19) is trivial. Moreover, we have

$$
H_{q-1}\left(\mathbb{T}\left(V_{\leqslant n}\right)\right)=\mathbb{T}_{q-1}\left(V_{\leqslant n}\right), \quad H_{q-1}(\alpha)=\alpha_{q-1} .
$$

As a result the group $\mathcal{D}_{n}^{q}$ consists of those pairs $(\xi, \alpha) \in \operatorname{aut}\left(V_{q}\right) \times \operatorname{aut}\left(\mathbb{T}\left(V_{\leqslant n}\right)\right)$ making the following diagram commute (see Definition 4.8):

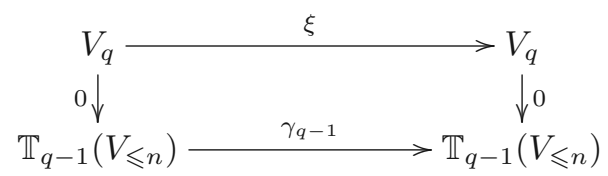

Therefore $\mathcal{D}_{n}^{q}$ is just the group $\operatorname{aut}\left(V_{q}\right) \times \operatorname{aut}\left(\mathbb{T}\left(V_{\leqslant n}\right)\right)$ used in the previous section.

Proposition 4.10. The map g: $\mathcal{E}\left(\mathbb{T}\left(V_{q} \oplus V_{\leqslant n}\right)\right) \rightarrow \mathcal{D}_{n}^{q}$ given by

$$
\mathrm{g}([\alpha])=\left(\widetilde{\alpha}_{q},\left[\alpha_{n}\right]\right)
$$

is a surjective homomorphism of groups.

Proof. First it is well known that if two chain morphisms are homotopic, then they induce the same graded linear maps on the chain complex of indecomposables, i.e., $\widetilde{\alpha}=\widetilde{\alpha^{\prime}}$. Moreover, $\alpha_{n}, \alpha_{n}^{\prime}$ are homotopic and, using the diagram (17), we deduce that the map $\mathrm{g}$ is well defined.

Next let $\left(\xi,\left[\alpha_{n}\right]\right) \in \mathcal{D}_{n}^{q}$. Recall that, in the diagram (20), we have:

$$
\begin{aligned}
b_{q}(v) & =\alpha_{n} \circ \partial(v)+\operatorname{Im} \partial_{\leqslant n}, \\
b_{q} \circ \xi_{q}(v) & =\partial \circ \xi(v)+\operatorname{Im} \partial_{\leqslant n},
\end{aligned}
$$

where $\partial_{\leqslant n}: \mathbb{T}_{q}\left(V_{\leqslant n}\right) \rightarrow \mathbb{T}_{q-1}\left(V_{\leqslant n}\right)$.

Since by Definition 4.8 this diagram commutes, the element $\left(\alpha_{n} \circ \partial-\partial \circ \xi\right)(v) \in$ 
$\operatorname{Im} \partial_{\leqslant n}$. As a consequence there exists $u_{v} \in \mathbb{T}_{q}\left(V^{\leqslant n}\right)$ such that

$$
\left(\alpha_{n} \circ \partial-\partial \circ \xi\right)(v)=\partial_{\leqslant n}\left(u_{v}\right) \text {. }
$$

Thus we define $\alpha$ : $\left(\mathbb{T}\left(V_{q} \oplus V_{\leqslant n}\right), \partial\right) \rightarrow\left(\mathbb{T}\left(V_{q} \oplus V_{\leqslant n}\right), \partial\right)$ by setting

$$
\alpha(v)=\xi(v)+u_{v} \quad \text { and } \quad \alpha=\alpha_{n} \text { on } V_{\leqslant n} .
$$

As $\partial(v) \in \mathbb{T}_{q-1}\left(V_{\leqslant n}\right)$ then, by $(21)$, we get

$$
\partial \circ \alpha(v)=\partial(\xi(v))+\partial_{n}\left(u_{v}\right)=\alpha_{n} \circ \partial(v)=\alpha \circ \partial(v) .
$$

So $\alpha$ is a chain algebra morphism. Now as $u_{v} \in \mathbb{T}_{q}\left(V_{\leqslant n}\right)$ and $q>n$, the homomorphism $\widetilde{\alpha}_{q}: V_{q} \rightarrow V_{q}$ coincides with $\xi$.

Then it is well known (see $[\mathbf{1}, \mathbf{9}]$ and $[\mathbf{1 0}]$ ) that any chain algebra morphism between two 1-connected chain algebras inducing a graded isomorphism on the homology of the chain complex of indecomposables (see 18) is a homotopy equivalence. Consequently, $[\alpha] \in \mathcal{E}(\mathbb{T}(V))$. Therefore $\mathrm{g}$ is onto.

Finally, the following relations

$$
\begin{aligned}
\mathrm{g}\left([\alpha]\left[\alpha^{\prime}\right]\right) & =\mathrm{g}\left(\left[\alpha \circ \alpha^{\prime}\right]\right)=\left(\widetilde{\alpha \circ \alpha^{\prime}}{ }_{q},\left[\alpha_{n} \circ \alpha_{n}^{\prime}\right]\right) \\
& =\left(\widetilde{\alpha}_{q},\left[\alpha_{n}\right]\right) \circ\left({\widetilde{\alpha^{\prime}}}_{q},\left[\alpha_{n}^{\prime}\right]\right)=\mathrm{g}([\alpha]) \circ \mathrm{g}\left(\left[\alpha^{\prime}\right]\right)
\end{aligned}
$$

assure that $\mathrm{g}$ is a homomorphism of groups.

\subsection{Characterization of ker g}

Next by definition we have:

$$
\text { ker } \mathrm{g}=\left\{[\alpha] \in \mathcal{E}\left(\mathbb{T}\left(V_{q} \oplus V_{\leqslant n}\right)\right) \mid \widetilde{\alpha}_{q}=i d_{V_{q}},\left[\alpha_{n}\right]=\left[i d_{\mathbb{T}\left(V_{\leqslant n}\right)}\right]\right\},
$$

therefore for every $[\alpha] \in$ ker g we have:

$$
\begin{aligned}
\alpha(v) & =v+z, \quad z \in \mathbb{T}_{q}\left(V_{\leqslant n}\right), \\
\alpha_{n} & \simeq i d_{\mathbb{T}\left(V_{\leqslant n}\right)} .
\end{aligned}
$$

So define:

$$
\theta_{\alpha}: V_{q} \rightarrow \mathbb{T}_{q}\left(V_{\leqslant n}\right) \quad \text { by } \theta_{\alpha}(v)=\alpha(v)-v .
$$

Notice that the relations (22) and (23) imply that

$$
\theta_{\alpha^{\prime} \circ \alpha}=\theta_{\alpha^{\prime}}+\theta_{\alpha} \text {. }
$$

Remark 4.11. If the differential in the chain algebra $\left(\mathbb{T}\left(V_{q} \oplus V_{\leqslant n}\right)\right)$ is trivial, then according to Remark 4.5 the formula (22) becomes

$$
\begin{aligned}
\alpha(v) & =v+z, \quad z \in \mathbb{T}_{q}\left(V_{\leqslant n}\right), \\
\alpha_{n} & =i d_{\mathbb{T}\left(V_{\leqslant n}\right)}
\end{aligned}
$$

implying that the element $\theta_{\alpha}(v)=z$ is a cycle in $\mathbb{T}_{q}\left(V_{\leqslant n}\right)$. Notice that if the differential is not trivial, then $\theta_{\alpha}(v)$ need not be a cycle. However, we have the following crucial lemma:

Lemma 4.12. Let $[\alpha] \in$ ker g. Then there exists $[\beta] \in$ ker g satisfying:

1. $\theta_{\beta}(v)$ is a cycle in $\mathbb{T}_{q}\left(V_{\leqslant n}\right)$ for every $v \in V_{q}$ 

2. $\beta_{n}=i d_{\mathbb{T}\left(V_{\leqslant n}\right)}$
3. $[\beta]=[\alpha]$

Proof. Write $V=V_{q} \oplus V_{\leqslant n}$. Since, $\left[\alpha_{n}\right]=\left[i d_{\mathbb{T}\left(V_{\leqslant n}\right)}\right]$ there is a homotopy:

$$
F:\left(\mathbb{T}\left(V_{\leqslant n}^{\prime} \oplus V_{\leqslant n}^{\prime \prime} \oplus(s V)_{\leqslant n}\right), D\right) \rightarrow\left(\mathbb{T}\left(V_{\leqslant n}\right), \partial\right)
$$

such that for every $x \in \mathbb{T}(V)$ we have

$$
F \circ i^{\prime}(x)=\alpha(x), \quad F \circ i^{\prime \prime}(x)=x .
$$

Thus we define $\beta$ by setting:

$$
\beta(v)= \begin{cases}\alpha(v)-F(S(\partial v)), & \text { for } v \in V_{q} \\ v, & \text { for } v \in V_{\leqslant n}\end{cases}
$$

Notice that as $v \in V_{q}$, we deduce that $\partial v \in \mathbb{T}_{q-1}(V)$. It follows that $S(\partial v) \in \mathbb{T}_{q}\left(V_{\leqslant n}^{\prime} \oplus\right.$ $\left.V_{\leqslant n}^{\prime \prime} \oplus(s V) \leqslant n\right)$, so the element $F(S(\partial v)) \in \mathbb{T}_{q}\left(V_{\leqslant n}\right)$.

Let us prove that $\beta$ is a chain algebra morphism. Indeed first, for $v \in V_{q}$, using the relations (14), we deduce that

$$
0=D^{2}(s v)=D\left(v^{\prime \prime}-v^{\prime}\right)-D S(\partial v) .
$$

Now by virtue of (25) and (26) we get

$$
\begin{aligned}
\partial(\beta(v)) & =\partial \circ \alpha(v)-\partial \circ F(S(\partial v)) \\
& =\partial \circ \alpha(v)-F(D S(\partial v)) \\
& =\partial \circ \alpha(v)-F\left(D\left(v^{\prime}-v^{\prime \prime}\right)\right) \\
& =\partial \circ \alpha(v)-F \circ i^{\prime}(\partial v)+F \circ i^{\prime \prime}(\partial v) \\
& =\partial \circ \alpha(v)-\alpha \circ \partial(v)+\partial(v) \\
& =\partial(v)=\beta(\partial(v)) .
\end{aligned}
$$

Here we use $(27)$ and the fact that $\partial(v) \in \mathbb{T}\left(V_{\leqslant n}\right)$ and $\beta$ is the identity on $V_{\leqslant n}$.

Consequently,

$$
\partial\left(\theta_{\beta}(v)\right)=\partial(\beta(v)-v)=\partial(\beta(v))-\partial(v)=\partial(v)-\partial(v)=0 .
$$

Thus $\theta_{\beta}(v)$ is a cycle in $\mathbb{T}_{q}\left(V_{\leqslant n}\right)$ and $\beta_{n}=i d_{\mathbb{T}\left(V_{\leqslant n}\right)}$. Next let us define

$$
G:\left(\mathbb{T}\left(V^{\prime} \oplus V^{\prime \prime} \oplus(s V)\right), D\right) \rightarrow(\mathbb{T}(V), \partial)
$$

by setting

$$
\begin{aligned}
G\left(v^{\prime}\right) & =\alpha(v), & & \text { on } V_{q}^{\prime}, \\
G\left(v^{\prime}\right) & =\beta(v), & & \text { on } V_{q}^{\prime \prime}, \\
G(s v) & =0, & & \text { on }(s V)_{q}, \\
G & =F, & & \text { on } V_{\leqslant n}^{\prime} \oplus V_{\leqslant n}^{\prime \prime} \oplus(s V)_{\leqslant n} .
\end{aligned}
$$


Using (25) and (28), an easy computation shows

$$
\begin{aligned}
& \partial \circ G\left(v^{\prime}\right)=\partial(\alpha(v)), \quad G \circ D\left(v^{\prime}\right)=G \circ i^{\prime}(\partial v)=F \circ i^{\prime}(\partial v)=\alpha(\partial v), \\
& \partial \circ G\left(v^{\prime \prime}\right)=\partial(\beta(v))=\partial v, \quad G \circ D\left(v^{\prime \prime}\right)=G \circ i^{\prime \prime}(\partial v)=F \circ i^{\prime \prime}(\partial v)=\partial v \text {, } \\
& \partial \circ G(s v)=0 \text {, } \\
& G \circ D(s v)=G \circ\left(v^{\prime}-v^{\prime \prime}-S(\partial v)\right) \\
& =G\left(v^{\prime}\right)-G\left(v^{\prime \prime}\right)-G(S(\partial v)) \\
& =\alpha(v)-\beta(v)-G(S(\partial v)) \\
& =F(S(\partial v))-G(S(\partial v)) \\
& =0 \text {. }
\end{aligned}
$$

Here we use the facts that $S(\partial v) \in \mathbb{T}_{q}\left(V_{\leqslant n}^{\prime} \oplus V_{\leqslant n}^{\prime \prime} \oplus(s V)_{\leqslant n}\right)$ and $G$ and $F$ coincide on $V_{\leqslant n}^{\prime} \oplus V_{\leqslant n}^{\prime \prime} \oplus(s V) \leqslant n$.

Finally, it easy to check (again by using (14)) that $G \circ i^{\prime}(v)=\alpha(v)$ and $G \circ i^{\prime \prime}(v)=$ $\beta(v)$ implying that $[\beta]=[\alpha]$.

Thus Lemma 4.12 and the relation (23) allow us to define a map

$$
\Phi: \operatorname{ker} \mathrm{g} \rightarrow \operatorname{Hom}\left(V_{q}, H_{q}\left(\mathbb{T}\left(V_{\leqslant n}\right)\right)\right)
$$

by setting $\Phi([\beta])(v)=\left\{\theta_{\beta}(v)\right\}$ for $v \in V_{q}$ where $[\beta]$ is chosen as in Lemma 4.12.

Proposition 4.13. The map $\Phi$ is an isomorphism.

Proof. Assume that $\Phi([\beta])(v)=\Phi\left(\left[\beta^{\prime}\right]\right)(v)$ in $H_{q}\left(\mathbb{T}\left(V_{\leqslant n}\right)\right)$, then $\theta_{\beta^{\prime}}(v)-\theta_{\beta}(v)=$ $\beta(v)-\beta^{\prime}(v)$ is a boundary and Lemma 4.4 implies that $[\beta]=\left[\beta^{\prime}\right]$. Hence $\Phi$ is one to one.

Given a homomorphism $\chi \in \operatorname{Hom}\left(V_{q}, H_{q}\left(\mathbb{T}\left(V_{\leqslant n}\right)\right)\right)$ and write $\chi(v)=\{\widetilde{\chi(v)}\}$, where $\widetilde{\chi(v)}$ is a cycle. We define $\beta:(\mathbb{T}(V), \partial) \rightarrow(\mathbb{T}(V), \partial)$ by:

$$
\beta(v)=v+\widetilde{\chi(v)} \text { for } v \in V_{q} \quad \text { and } \quad \beta=i d \quad \text { on } V_{\leqslant n} .
$$

Then $\beta$ is a chain algebra morphism with $\Phi([\beta])=\chi$. Hence $\Phi$ is onto.

Finally, given $\beta, \beta^{\prime} \in \operatorname{kerg}$ as in Lemma 4.12. So $\beta(v)=v+\theta_{\beta}(v)$ and $\beta^{\prime}(v)=$ $v+\theta_{\beta^{\prime}}(v)$ for $v \in V_{q}$. Therefore by (24) we get:

$$
\beta^{\prime} \circ \beta(v)=v+\theta_{\beta^{\prime}}(v)+\theta_{\beta}(v)=v+\theta_{\beta^{\prime} \circ \beta}(v) .
$$

Consequently, $\Phi\left(\left[\beta^{\prime}\right] .[\beta]\right)=\Phi\left(\left[\beta^{\prime} \circ \beta\right]\right)=\theta_{\beta^{\prime} \circ \beta}=\theta_{\beta^{\prime}}+\theta_{\beta}=\Phi\left(\left[\beta^{\prime}\right]\right)+\Phi([\beta])$. Thus $\Phi$ is a homomorphism of groups.

Summarizing, we have proven:

Theorem 4.14. Let $\left(\mathbb{T}\left(V_{q} \oplus V_{\leqslant n}\right), \partial\right)$ be a 1-connected chain algebra. Then there exists a short exact sequence of groups

$$
\operatorname{Hom}\left(V_{q}, H_{q}\left(\mathbb{T}\left(V_{\leqslant n}\right)\right)\right) \longmapsto \mathcal{E}\left(\mathbb{T}\left(V_{q} \oplus V_{\leqslant n}\right)\right) \rightarrow \mathcal{D}_{n}^{q} .
$$

We now focus on the subgroup $\mathcal{E}_{*}\left(\mathbb{T}\left(V_{q} \oplus V_{\leqslant n}\right)\right)$ of $\mathcal{E}\left(\mathbb{T}\left(V_{q} \oplus V_{\leqslant n}\right)\right)$ formed of the elements inducing the identity on the graded homology module $H_{*}(V, d)$. Let us define $\mathcal{G}_{n}^{q}$ as the subgroup of $\mathcal{E}_{*}\left(\mathbb{T}\left(V_{\leqslant n}\right)\right)$ consisting of the elements $[\alpha]$ satisfying $H_{q-1}(\alpha) \circ b_{q}=b_{q}$ where $b_{q}: V_{q} \rightarrow H_{q-1}\left(\mathbb{T}\left(V_{\leqslant n}\right)\right)$ is as in $(16)$.

Theorem 4.15. Let $q>n$ and let $\left(\mathbb{T}\left(V_{q} \oplus V_{\leqslant n}\right), \partial\right)$ be a 1-connected chain algebra. Then there exists a short exact sequence of groups

$$
\operatorname{Hom}\left(V_{q}, H_{q}\left(\mathbb{T}\left(V_{\leqslant n}\right)\right)\right) \longmapsto \mathcal{E}_{*}\left(\mathbb{T}\left(V_{q} \oplus V_{\leqslant n}\right)\right) \rightarrow \mathcal{G}_{n}^{q} .
$$


Proof. First let $[\beta] \in$ ker g. Lemma 4.12 assures that $\widetilde{\alpha}_{q}=i d_{V_{q}}$ and $\alpha_{n}=i d_{\mathbb{T}\left(V_{\leqslant n}\right)}$, therefore $\widetilde{\alpha}=i d_{V}$. It follows that ker $\mathrm{g} \subseteq \mathcal{E}_{*}\left(\mathbb{T}\left(V_{q} \oplus V_{\leqslant n}\right)\right)$.

Next from (29) we obtain

$$
\mathrm{g}\left(\mathcal{E}_{*}\left(\mathbb{T}\left(V_{q} \oplus V_{\leqslant n}\right)\right)\right)=\left\{\Psi([\alpha])=\left(\widetilde{\alpha}_{q},\left[\alpha_{n}\right]\right) \mid[\alpha] \in \mathcal{E}_{*}\left(\mathbb{T}\left(V_{q} \oplus V_{\leqslant n}\right)\right)\right\} .
$$

As $[\alpha] \in \mathcal{E}_{*}\left(\mathbb{T}\left(V_{q} \oplus V_{\leqslant n}\right)\right)$, the graded automorphism $H_{*}(\widetilde{\alpha})$ is the identity which, in turn, implies that $\widetilde{\alpha}_{q}=\operatorname{id}_{V^{q}}$ and, as the pair $\left(\operatorname{id}_{V^{q}},\left[\alpha_{n}\right]\right)$ makes the diagram (17) commute, we can identify $\mathrm{g}\left(\mathcal{E}_{\sharp}\left(\Lambda\left(V^{q} \oplus V^{\leqslant n}\right)\right)\right)$ with the subgroup $\mathcal{G}_{n}^{q}$.

Corollary 4.16. Let $\left(\mathbb{T}\left(V_{q} \oplus V_{\leqslant n}\right), \partial\right)$ be a 1-connected chain algebra. If $\mathcal{E}_{*}\left(\mathbb{T}\left(V_{\leqslant n}\right)\right)$ is trivial, then:

$$
\operatorname{Hom}\left(V_{q}, H_{q}\left(\mathbb{T}\left(V_{\leqslant n}\right)\right)\right) \cong \mathcal{E}_{*}\left(\mathbb{T}\left(V_{q} \oplus V_{\leqslant n}\right)\right) .
$$

Corollary 4.17. Let $\left(\mathbb{T}\left(V_{2 n} \oplus V_{\leqslant 2 n-1}\right), \partial\right)$ be an $n$-connected chain algebra, i.e., $V_{k}=0$ for $k<n$. Then

$$
\operatorname{Hom}\left(V_{2 n}, V_{n} \otimes V_{n}\right) \cong \mathcal{E}_{*}\left(\mathbb{T}\left(V_{2 n} \oplus V_{\leqslant 2 n-1}\right)\right) .
$$

Proof. First, as $\left(\mathbb{T}\left(V_{2 n} \oplus V_{\leqslant 2 n-1}\right), \partial\right)$ is $n$-connected, the group $\mathcal{E}_{*}\left(\mathbb{T}\left(V_{\leqslant 2 n-1}\right)\right)$ is trivial. Next clearly $H_{2 n}\left(\mathbb{T}\left(V_{\leqslant 2 n-1}\right)\right)=V_{n} \otimes V_{n}$, hence (30) follows from Corollary 4.16.

Corollary 4.18. Let $\left(\mathbb{T}\left(V_{q} \oplus V_{\leqslant n}\right), \partial\right)$ be a 1-connected chain algebra. If the group $\mathcal{E}\left(\mathbb{T}\left(V_{q} \oplus V_{\leqslant n}\right)\right)$ is finite, then the linear map $b_{q}$ is injective.

Proof. Assume that $b_{q}$ is not injective and let $v_{0} \neq 0 \in V_{q}$ be such that $b_{q}\left(v_{0}\right)=0$. For every $a \neq 0 \in \mathbb{Q}$, we define $\xi_{a}: V_{q} \rightarrow V_{q}$ by

$$
\xi\left(v_{0}\right)=a v_{0}, \quad \xi_{a}=i d \quad \text { otherwise. }
$$

Clearly the pair $\left(\xi_{a},[i d]\right) \in \operatorname{aut}\left(V_{q}\right) \times \mathcal{E}\left(\mathbb{T}\left(V_{\leqslant n}\right)\right)$ for every $a \neq 0 \in \mathbb{Q}$ and makes following diagram commute

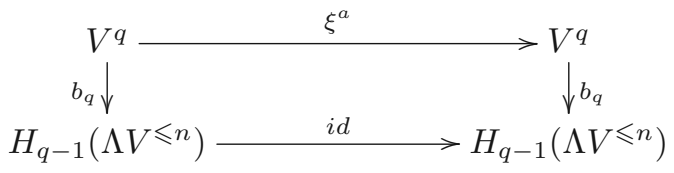

Therefore $\left(\xi_{a},[i d]\right) \in \mathcal{D}_{n-1}^{q}$ for every $a \neq 0 \in \mathbb{Q}$ implying that the group $\mathcal{D}_{n}^{q}$ is infinite. Consequently, the group $\mathcal{E}\left(\mathbb{T}\left(V_{q} \oplus V_{\leqslant n}\right)\right)$ is also infinite according the exact sequence (29).

\section{4. $\quad r$-Mild differential graded Lie algebras}

Let $R \subseteq \mathbb{Q}$ be a ring such that, for some prime $p, R$ contains $n^{-1}$ for $n<p$. A free differential graded Lie algebra $(\mathbb{L}(V), \partial)$ over $R$ is called $r$-mild if

$$
V_{k}=0, \quad k \leqslant r-1, \quad k \geqslant p r-1 .
$$

Recall that, in [1], Anick defined a reasonable concept of "homotopy" among morphisms between free $R$-dgls, analogous in many respects to the topological notion of homotopy and proved the following result: 
Theorem 4.19 ([1] proposition 3.3). Let $f, g:(\mathbb{L}(V), \partial) \rightarrow(\mathbb{L}(W), \partial)$ be two r-mild free differential graded Lie algebras. Then $f, g$ are homotopic as dgl-morphisms if and only if $U f, U g$ are homotopic as chain algebra morphisms.

Proposition 4.20. Let $(\mathbb{L}(V), \partial)$ be an $r$-mild free differential graded Lie algebra. For any $r \leqslant n<q<r p$, the homomorphism

$$
\phi_{n}^{q}: \mathcal{E}\left(\mathbb{L}\left(V_{q} \oplus V_{\leqslant n}\right)\right) \rightarrow \mathcal{E}\left(\mathbb{T}\left(V_{q} \oplus V_{\leqslant n}\right)\right), \quad \phi_{n}^{q}([\alpha])=[U(\alpha)]
$$

is injective.

Proof. First, it is well known that if $\alpha$ is equivalence of homotopy, then so is $U(\alpha)$, hence $\phi_{n}^{q}$ is well defined.

Next if $U(\alpha) \simeq i d_{\mathbb{T}\left(V_{q} \oplus V_{\leqslant n}\right)}$, then from Theorem 4.19 we deduce that $\alpha \simeq$ $i d_{\mathbb{L}\left(V_{q} \oplus V_{\leqslant n}\right)}$. Therefore $\phi_{n}^{q}$ is injective.

Using the same argument we can deduce

Corollary 4.21. Let $(\mathbb{L}(V), \partial)$ be an r-mild free differential graded Lie algebra. For any $r \leqslant n<q<r p$, the homomorphism

$$
\psi_{n}^{q}: \mathcal{E}_{*}\left(\mathbb{L}\left(V_{q} \oplus V_{\leqslant n}\right)\right) \rightarrow \mathcal{E}_{*}\left(\mathbb{T}\left(V_{q} \oplus V_{\leqslant n}\right)\right), \quad \psi_{n}^{q}([\alpha])=[U(\alpha)]
$$

is injective.

\section{Topological applications}

Let $X$ be a simply connected CW-complex of dimension $n+1$. For $q>n$ let

$$
Y=X \cup_{\alpha}\left(\bigcup_{i \in I} e_{i}^{q+1}\right)
$$

be the space obtained by attaching cells of dimension $q+1$ to $X$ by a map $\alpha$ : $\underset{i \in I}{\vee}$ $\mathbb{S}^{q+1} \rightarrow X$.

Recall that the Adams-Hilton model of $Y$ is a chain algebra morphism

$$
\Theta_{Y}:\left(\mathbb{T}\left(V_{q} \oplus V_{\leqslant n}\right), \partial\right) \rightarrow C_{*}(\Omega Y, R)
$$

such that

$$
H_{*}\left(\Theta_{Y}\right): H_{*}\left(\mathbb{T}\left(V_{q} \oplus V_{\leqslant n}\right), \partial\right) \rightarrow H_{*}(\Omega Y, R)
$$

is an isomorphism of graded algebras and such that

$$
H_{i-1}\left(V_{q} \oplus V_{\leqslant n}, d\right) \cong H_{i}(Y, R), \quad \text { as graded modules. }
$$

Here $C_{*}(\Omega Y, R)$ denotes the complex of non-degenerate cubic chains equipped with the multiplication induced by composition of loops. We denote by $A(Y)$ the chain algebra $\left(\mathbb{T}\left(V_{q} \oplus V_{\leqslant n}\right), \partial\right)$.

Notice also that the free module $V_{i}$ admits a basis consisting of the cells of dimension $i+1$ of $Y$ and the differential $\partial$ is determined by the attaching maps of the cells (see for example [1] for more details). Consequently, we have

$$
V_{q} \cong H_{q+1}(Y, X ; R)
$$

where $H_{q+1}(Y, X ; R)$ denotes the free $R$-module of the homology of the pair $(Y, X)$ in degree $q+1$. 
By the properties of the Adams-Hilton model, the chain algebra $\left(\mathbb{T}\left(V_{\leqslant n}\right), \partial\right)$ may be considered as the Adams-Hilton model of $X$, i.e., $A(X)=\left(\mathbb{T}\left(V_{\leqslant n}\right), \partial\right)$. Moreover, if $[f] \in \mathcal{E}(A(X))$, then $f$ induces the following commutative diagram

$$
\begin{array}{rr}
H_{q-1}\left(\mathbb{T}\left(V_{\leqslant n}\right)\right) \stackrel{H_{q-1}(f)}{ } & H_{q-1}\left(\mathbb{T}\left(V_{\leqslant n}\right)\right) \\
\cong \downarrow H_{q-1}\left(\Theta_{X}\right) & \cong H_{q-1}\left(\Theta_{X}\right) \\
H_{q-1}(\Omega X, R) \stackrel{H_{q-1}\left(\Theta_{X}\right) \circ H_{q-1}(f) \circ\left(H_{q-1}\left(\Theta_{X}\right)\right)^{-1}}{\longrightarrow} & H_{q-1}(\Omega X, R)
\end{array}
$$

Definition 5.1. Set $\widetilde{H}_{q-1}(f)=H_{q-1}\left(\Theta_{X}\right) \circ H_{q-1}(f) \circ\left(H_{q-1}\left(\Theta_{X}\right)\right)^{-1}$. We define $\Gamma_{n}^{q+1}$ to be the subset of aut $\left(H_{q+1}(Y ; R)\right) \times \mathcal{E}(A(X))$ consisting of the pairs $(\xi,[f])$ making the following diagram commute

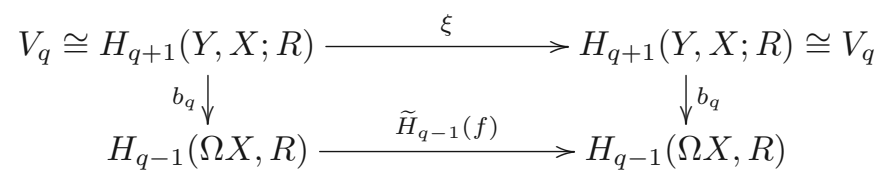

and

$$
\Pi_{n}^{q+1}=\left\{[f] \in \mathcal{E}_{*}(A(X)) \mid \widetilde{H}_{q-1}(f) \circ b_{q}=b_{q}\right\} .
$$

Clearly $\Gamma_{n}^{q+1}$ is a subgroup of $\operatorname{aut}\left(H_{q+1}(Y, X ; R)\right) \times \mathcal{E}(A(X))$ and $\Pi_{n}^{q+1}$ is a subgroup of $\mathcal{E}_{*}(A(X))$.

Remark 5.2. It is important to notice that if the homomorphism $b_{q}$ is trivial, then

$$
\Gamma_{n}^{q+1}=\operatorname{aut}\left(H_{q+1}(Y, X ; R)\right) \times \mathcal{E}(A(X)), \quad \Pi_{n}^{q+1}=\mathcal{E}_{*}(A(X))
$$

and if $b_{q}$ is an isomorphism, then from the commutative diagram (30) we deduce that $\xi=\left(b_{q}\right)^{-1} \circ H_{q-1}(f) \circ b_{q}$. Therefore the map

$$
\mathcal{E}(A(X)) \rightarrow \Gamma_{n}^{q+1}, \quad[f] \longmapsto\left(\left(b_{q}\right)^{-1} \circ H_{q-1}(f) \circ b_{q},[\alpha]\right)
$$

is an isomorphism. In this case, if $[f] \in \Pi_{n}^{q+1}$, then $\widetilde{H}_{q-1}(f) \circ b_{q}=b_{q}$ and as $b_{q}$ is an isomorphism it follows that $\widetilde{H}_{q-1}(f)=i d$. Consequently,

$$
\Pi_{n}^{q+1}=\left\{[f] \in \mathcal{E}_{*}(A(X)) \mid \widetilde{H}_{q-1}(f)=i d\right\} .
$$

Theorem 5.3. Let $X$ be a simply connected $C W$-complex of dimension $n+1$ and let $Y$ be as in (31). Then there exist two short exact sequences of groups

$$
\oplus_{i} H_{q}(\Omega X, R) \longmapsto \mathcal{E}(A(Y)) \rightarrow \Gamma_{n}^{q+1}, \quad \oplus_{i} H_{q}(\Omega X, R) \longmapsto \mathcal{E}_{*}(A(Y)) \rightarrow \Pi_{n}^{q+1} .
$$

Proof. The two sequences (32) follow from a mere transcription of Theorems 4.14 and 4.15 in the topological context using the properties of the Adams-Hilton model. Note that this model implies the identifications $\Gamma_{n}^{q+1} \cong \mathcal{D}_{n}^{q}$ and $\Pi_{n}^{q+1} \cong \mathcal{G}_{n}^{q}$.

Combining Remark 5.2 and Theorem 5.3 we derive the following results: 
Corollary 5.4. Let $X$ be a simply connected $C W$-complex of dimension $n+1$ and let $Y$ be as in (31). If the homomorphism $b_{q}: H_{q+1}(Y, X ; R) \rightarrow H_{q-1}(\Omega X, R)$ is bijective, then there exist two short exact sequences of groups

$$
\begin{aligned}
\oplus_{i} H_{q}(\Omega X, R) & \longmapsto \mathcal{E}(A(Y)) \rightarrow \mathcal{E}(A(X)), \\
\oplus_{i} H_{q}(\Omega X, R) & \longmapsto \mathcal{E}_{*}(A(Y)) \rightarrow\left\{[f] \in \mathcal{E}_{*}(A(X)) \mid \widetilde{H}_{q-1}(f)=i d\right\} .
\end{aligned}
$$

Corollary 5.5. Let $X$ be a simply connected $C W$-complex of dimension $n+1$ and let $Y$ be as in (31). If the homomorphism $b_{q}: H_{q+1}(Y, X ; R) \rightarrow H_{q-1}(\Omega X, R)$ is trivial, then there exist two short exact sequences of groups

$$
\begin{aligned}
\oplus_{i} H_{q}(\Omega X, R) & \longmapsto \mathcal{E}(A(Y)) \rightarrow \operatorname{aut}\left(H_{q+1}(Y, X ; R)\right) \times \mathcal{E}(A(X)), \\
\oplus_{i} H_{q}(\Omega X, R) & \longmapsto \mathcal{E}_{*}(A(Y)) \rightarrow \mathcal{E}_{*}(A(X)) .
\end{aligned}
$$

As a consequence of Corollaries 5.4 and 5.5 we derive

Corollary 5.6. Let $X$ be a simply connected $C W$-complex of dimension $n+1$ and let $Y$ be as in (31).

If the homomorphism $b_{q}$ is trivial, then

$$
\frac{\mathcal{E}(A(Y))}{\mathcal{E}_{*}(A(Y))} \cong \operatorname{aut}\left(H_{q+1}(Y, X ; R)\right) \times \frac{\mathcal{E}(A(X))}{\mathcal{E}_{*}(A(X))}
$$

If $b_{q}$ is an isomorphism, then

$$
\frac{\mathcal{E}(A(Y))}{\mathcal{E}_{*}(A(Y))} \cong \frac{\mathcal{E}(A(X))}{\left\{[f] \in \mathcal{E}_{*}(A(X)) \mid \widetilde{H}_{q-1}(f)=i d\right\}} .
$$

\subsection{Anick model}

We assume that $R \subseteq \mathbb{Q}$ is a ring with least non-invertible prime $p>2$. With $R$ fixed, we take $1 \leqslant r<k$ satisfying $k<\min (r+2 p-3$, rp -1$)$. When $R=\mathbb{Q}$ we assume $r=1$ and $k$ is infinite.

Let $X$ be an $r$-connected finite CW-complex of dimension $n+1 \leqslant k$. For $k \geqslant q>$ $n+1$, let

$$
Y=X \cup_{\alpha}\left(\bigcup_{i \in I} e_{i}^{q+1}\right)
$$

as in (31). Recall that the Anick model of $Y$ (see $[\mathbf{1}, \mathbf{2}]$ for more details) is a free differential graded Lie algebra $\left(\mathbb{L}\left(V_{q} \oplus V_{\leqslant n}\right), \partial\right)$ over $R$ such that

$$
H_{*-1}\left(\mathbb{L}\left(V_{q} \oplus V_{\leqslant n}\right), \partial\right) \cong \pi_{*}(Y,) \otimes R, \quad H_{*-1}\left(V_{q} \oplus V_{\leqslant n}, d\right) \cong H_{*}(Y, R) .
$$

Moreover, by properties of this model we deduce

$$
\mathcal{E}_{*}\left(\mathbb{L}\left(V_{q} \oplus V_{\leqslant n}\right)\right) \cong \mathcal{E}_{*}\left(X_{R}\right), \quad \mathcal{E}\left(\mathbb{L}\left(V_{q} \oplus V_{\leqslant n}\right)\right) \cong \mathcal{E}\left(X_{R}\right)
$$

Here $X_{R}$ denotes the $R$-localization of $X$. From Proposition 4.20 and Corollary 4.21 we derive the following result 
Theorem 5.7. Let $Y$ be the space in (33). The homomorphisms

$$
\mathcal{E}\left(Y_{R}\right) \rightarrow \mathcal{E}\left(A\left(Y_{R}\right)\right), \quad \mathcal{E}_{*}\left(Y_{R}\right) \rightarrow \mathcal{E}_{*}\left(A\left(Y_{R}\right)\right)
$$

are injective.

\section{References}

[1] D.J. Anick, Hopf algebras up to homotopy, J. Amer. Math. Soc. (1989), 2(3): 417-452.

[2] D.J. Anick, An R-local Milnor-Moore theorem, Adv. Math. (1989), 77: 116-136.

[3] W. Barcus and M.G. Barratt, On the homotopy classification of the extensions of a fixed map, Trans. Amer. Math. Soc. (1958), 88: 57-74.

[4] H.J. Baues, Homotopy type and homology, Oxford Math. Monogr., Oxford University Press, New York, 1996.

[5] M. Benkhalifa, On the group of self-homotopy equivalences of $n$-connected and $(3 n+2)$-dimensional CW-Complex, Topology Appl. (2018), 233: 1-15.

[6] M. Benkhalifa, Postnikov decomposition and the group of self-equivalences of a rationalized space, Homology Homotopy Appl. (2017), 19(1): 209-224.

[7] M. Benkhalifa, Realizability of the group of rational self-homotopy equivalences, J. Homotopy Relat. Struct. (2011), 5(1): 361-372.

[8] M. Benkhalifa, Rational self-homotopy equivalences and Whitehead exact sequence, J. Homotopy Relat. Struct. (2009), 4: 111-121.

[9] M. Benkhalifa, Whitehead exact sequence and differential graded free Lie algebra, J. Math. (2004), 10: 987-1005.

[10] M. Benkhalifa, On the homotopy type of a chain algebra, Homology Homotopy Appl. (2004), 6(1): 109-135.

[11] M. Benkhalifa and S.B. Smith, The effect of cell attachment on the group of self-equivalences of an R-local space, J. Homotopy Relat. Struct. (2015), 4(1): $135-144$.

[12] R. Bott and H. Samelson, On the Pontryagin product in spaces of paths, Comment. Math. Helv. (1953), 27(1): 320-337.

[13] P.J. Kahn, Self-equivalences of $(n-1)$-connected $2 n$-manifolds, Bull. Amer. Math. Soc. (1966), 72: 562-566.

[14] S. Oka, N. Sawashita and M. Sugawara, On the group of self-equivalences of a mapping cone, Hiroshima Math. J. (1974), 4: 9-28.

[15] J. Rutter, The group of homotopy self-equivalence classes of $C W$-complexes, Math. Proc. Cambridge Philos. Soc. (1983), 93: 275-293.

[16] J. Rutter, Spaces of homotopy self-equivalences: A survey, Lecture Notes in Math., vol. 162, Springer-Verlag, Berlin (1997).

Mahmoud Benkhalifa mbenkhalifa@sharjah.ac.ae

Department of Mathematics, Faculty of Sciences, University of Sharjah, Sharjah, United Arab Emirates 